\title{
Fetal-maternal complications and their association with gestational thrombocytopenia
}

\author{
Vesna Elveđi-Gašparović ${ }^{\text {, Petrana Beljan }}{ }^{1}$, Snježana Gverić-Ahmetašević ${ }^{2}$, \\ Snježana Schuster ${ }^{3}$, Snježana Škrablin ${ }^{1}$ \\ ${ }^{1}$ Department of Obstetrics and Gynecology, School of Medicine, University of Zagreb, Zagreb, Croatia \\ ${ }^{2}$ Department of Obstetrics and Gynecology, Neonatal Intensive Care Unit, School of Medicine, University of Zagreb, Zagreb, Croatia \\ ${ }^{3}$ University of Applied Health Studies, Zagreb, Croatia
}

\begin{abstract}
Objectives: Thrombocytopenia is defined as a platelet count of $<150 \times 10^{9} / \mathrm{L}$. It is a common hematologic abnormality during pregnancy. Evaluation and treatment of gestational thrombocytopenia can be both, expensive and invasive, and may result in an adverse outcome. The aim of our study was to investigate the incidence of perinatal complications in pregnancies complicated with gestational thrombocytopenia and to determine if the severity of maternal gestational thrombocytopenia implicates unfavorable perinatal outcome.

Material and methods: Over a period of three years, we identified 50 patients with gestational thrombocytopenia: 38 with platelet count between $50-100 \times 10^{9} / \mathrm{L}$ - classified as moderate thrombocytopenia, and 12 with platelet count of $<50 \times 10^{9} / \mathrm{L}$ - classified as severe thrombocytopenia. Fifty women with normal platelet count constituted the control group. Maternal complications and neonatal outcomes were compared.

Results: Neonatal thrombocytopenia occurred more often in pregnancies complicated with gestational thrombocytopenia $(p=0.041)$. Thrombocytopenia in previous pregnancy seems to be an important predicting factor for disease severity in the current pregnancy $(p=0.01)$.

Conclusions: Gestational thrombocytopenia, even if severe, is not associated with adverse maternal or neonatal outcome. Moderate neonatal thrombocytopenia is more common in pregnancies complicated with severe gestational thrombocytopenia. The incidence of severe gestational thrombocytopenia is higher in patients with thrombocytopenia in previous pregnancy.
\end{abstract}

Key words: gestational thrombocytopenia, neonatal thrombocytopenia, perinatal outcome, platelet count

Ginekologia Polska 2016; 87, 6: 454-459

\section{INTRODUCTION}

Thrombocytopenia affects $6.6-11.6 \%$ of all pregnancies [1]. It is the second most common hematologic abnormality during pregnancy but it is usually a benign condition. Thrombocytopenia in pregnancy can be isolated or associated with systemic disorders like severe preeclampsia, HELLP syndrome (hemolysis, elevated liver enzymes, low platelets), or AFLP (acute fatty liver of pregnancy) [1]. Furthermore, autoimmune diseases, including systemic lupus erythematosus, antiphospholipid syndrome, thrombotic thrombocytopenic purpura, hemolytic uremic syndrome, and immune thrombocytopenia (ITP) may relapse or be first detected during pregnancy.

Major mechanisms for thrombocytopenia are decreased production and increased destruction of platelets, platelet sequestration, and hemodilution. Thrombocytopenia may also be the primary manifestation of viral infections (HIV, EBV, CMV), or a common adverse reaction from many drugs (heparin, antibiotics, nonsteroidal anti-inflammatory drugs, diuretics). Magannet and Martin [2], classified thrombocytopenia according to its severity into: mild $\left(100\right.$ to $\left.<150 \times 10^{9} / \mathrm{L}\right)$, moderate $\left(50\right.$ to $\left.<100 \times 10^{9} / \mathrm{L}\right)$, 
and severe $\left(<50 \times 10^{9} / \mathrm{L}\right)$. There are no diagnostic tests for gestational thrombocytopenia. The disease is diagnosed by exclusion and is defined by the platelet count of no less than $70 \times 10^{9} / \mathrm{L}$, with the count returning to normal within 12 weeks after delivery [3].

Increased blood volume, increased platelet activation, and increased platelet clearance contribute to a'physiologic' decrease in platelet count during pregnancy [4]. Gestational thrombocytopenia is considered to be the most prevalent cause of thrombocytopenia in pregnancy and accounts for about $75 \%$ of cases its cases during pregnancy. The disease tends to occur late, usually during the third trimester $[5,6]$. Whereas it is commonly believed that the prognosis for mild thrombocytopenia is most often favorable, we decided to focus on moderate and severe thrombocytopenia and their impact on the pregnancy outcome.

Differentiating gestational thrombocytopenia from immune thrombocytopenia during pregnancy is clinically relevant, because pregnancies in women with immune thrombocytopenia may be complicated by severe neonatal thrombocytopenia, with a risk of neonatal intracranial hemorrhage $[7,8]$. Our study was carried out to investigate the incidence of perinatal complications in pregnancies complicated with gestational thrombocytopenia and to define if the severity of maternal gestational thrombocytopenia implicates unfavorable perinatal outcome.

\section{MATERIAL AND METHODS}

This retrospective case-control study was conducted at the Department of Obstetrics and Gynecology, School of Medicine, University of Zagreb. Data for the study were collected from all patients with gestational thrombocytopenia during a-3-year period (from January 2011 to December 2013). We analyzed the platelet count of all singleton deliveries after 28 weeks of gestation (according to the last menstrual period or first trimester fetal ultrasonography). Pregnancies complicated with fetal structural or chromosomal anomalies, preexisting maternal diseases (diabetes mellitus, liver or renal diseases, blood count diseases, immune thrombocytopenia known before pregnancy, systemic lupus erythematosus), and gestational hypertensive diseases (preeclampsia, HELLP syndrome, eclampsia) were excluded from the study.

Fifty women constituting the study group had negative anti-thrombocyte antibodies and gestational thrombocytopenia with the platelet count of $<100 \times 10^{9} / \mathrm{L}$. Out of them, 38 subjects had a platelet count between $50-100 \times 10^{9} / \mathrm{L}$ and were classified as having moderate thrombocytopenia. Twelve women from the study group had the platelet count of $<50 \times 10^{9} / \mathrm{L}$ and were classified as having severe thrombocytopenia. Fifty women with normal platelet count were recruited as controls. The control group included healthy pregnant women, matched for parity and gestational age with the study group.

The following characteristics were compared: mode of delivery, thrombocytopenia in previous pregnancy, infection during pregnancy (significant bacteriuria/microbiologically positive cervical smear), placental abruption, and severe postpartum bleeding. Fetal outcomes were compared with regard to birth weight, neonatal acidosis $(\mathrm{pH}$ from umbilical vein $<7.20$ ), Apgar scores at 1 and 5 minutes, admission to the Neonatal Intensive Care Unit (NICU), neonatal thrombocytopenia (platelet count of $<150 \times 10^{9} / \mathrm{L}$ ), intrauterine growth restriction (fetal weight below the $10^{\text {th }}$ percentile for gestational age), and stillbirth. Newborns weighing < 2500 grams were classified as 'low birth weight.' Platelet counts of all newborns were observed in the first 48 hours postpartum. Statistical analysis was performed using SPSS version 20 (SPSS Inc., Chicago, IL, USA). Nominal variables were expressed by count and percentage. Descriptive statistics were computed for continuous and categorical variables. Frequencies are presented for the categorical variables between the study groups. We used the two sample t-test for continuous variables. Categorical variables between the groups were analyzed using the $x^{2}$-test or Fisher's exact test. We used the logistic regression model to assess the independence of specific perinatal outcome parameters from prognostic factors. The logistic regression model was used to assess the independence of specific perinatal outcome parameters from the prognostic factors. The p-value of $<0.05$ was considered as statistically significant.

\section{RESULTS}

No differences in the proportion of term deliveries and gestational age at delivery were observed. Thrombocytopenia in pregnancy did not affect the mode of delivery and preterm delivery rate. Cesarean section was performed in 18 patients (36\%) from the study group and 11 patients (22\%) from the control group $(p=0.137)$ (Table 1).

Only 1 case of postpartum hemorrhage was found in the patient with severe gestational thrombocytopenia, and 1 case of placental abruption in the patient with moderate gestational thrombocytopenia (platelet count of $<83 \times 10^{9}$ ). Infection during pregnancy was significantly higher in the study group as compared to controls $(p=0.046$ ) (Table 2 ).

The incidence of neonatal thrombocytopenia was significantly higher in the study group $(p=0.041)$. Also, we found a higher admission rate to NICU ( $p=0.153)$, IUGR $(p=0.40)$, acidosis $(p=0.558)$, and low birth weight neonates $(p=0.081)$ in the study group (Figure 1$)$.

In the study group, 38 women had moderate gestational thrombocytopenia (platelet count of $50-100 \times 10^{9} / \mathrm{L}$ ) and 12 patients had severe gestational thrombocytopenia (platelet count of $<50 \times 10^{9} / \mathrm{L}$ ). Two patients with severe thrombocytopenia in the current pregnancy $(6.6 \%)$ had 


\begin{tabular}{|c|c|c|c|}
\hline Characteristics & $\begin{array}{l}\text { STUDY } \\
\text { group } \\
(n=50)\end{array}$ & $\begin{array}{l}\text { CONTROL } \\
\text { group } \\
(n=50)\end{array}$ & $P$-value \\
\hline Thrombocytopenia in previous pregnancy & $2(4 \%)$ & 0 & 0.153 \\
\hline Infection during pregnancy* & $8(16 \%)$ & $2(4 \%)$ & 0.046 \\
\hline Gestational age (week) & $38(33-41)$ & $39(36-42)$ & 0.634 \\
\hline Placental abruption & $1(2 \%)$ & 0 & 0.315 \\
\hline Preterm delivery & $5(10 \%)$ & $2(4 \%)$ & 0.240 \\
\hline Postpartum hemorrhage & $1(2 \%)$ & 0 & 0.315 \\
\hline Cesarean section & $18(36 \%)$ & $11(22 \%)$ & 0.137 \\
\hline Fetal demise in utero & $1(2 \%)$ & 0 & 0.315 \\
\hline
\end{tabular}

*Significant bacteriuria/microbiologically positive cervical smear; data are expressed as median or $\mathrm{n}(\%)$. Study group: platelet count $<100 \times 10^{9} / \mathrm{L}$. Control group: platelet count $>150 \times 10^{9} / \mathrm{L}$

\begin{tabular}{|c|c|c|c|}
\hline Outcome parameter & $\begin{array}{c}\text { STUDY } \\
\text { group } \\
(n=50)\end{array}$ & $\begin{array}{l}\text { CONTROL } \\
\text { group } \\
(n=50)\end{array}$ & $P$-value \\
\hline Umbilical vein $\mathrm{pH}<7.20$ & $2(4 \%)$ & 1 & 0.558 \\
\hline 1-min. Apgar score $<7$ & $2(4 \%)$ & $1(2 \%)$ & 0.558 \\
\hline 5-min. Apgar score $<7$ & $2(4 \%)$ & 0 & 0.153 \\
\hline Low birth weight $(<2500 \mathrm{~g}$ ) & $7(14 \%)$ & $2(4 \%)$ & 0.081 \\
\hline Neonatal thrombocytopenia & $4(8 \%)$ & 0 & 0.041 \\
\hline Admission to NICU & $2(4 \%)$ & 0 & 0.153 \\
\hline IUGR & $4(8 \%)$ & $2(4 \%)$ & 0.40 \\
\hline Neonatal death & 0 & 0 & - \\
\hline $\mathrm{ICH}$ & 0 & 0 & - \\
\hline
\end{tabular}

Data are expressed as median or $\mathrm{n}(\%)$. Study group: platelet count $<100 \times 10^{9} / \mathrm{L}$. Control group: platelet count $>150 \times 10^{9} / \mathrm{L}$. NICU - neonatal intensive care unit; IUGR - intrauterine growth restriction; ICH - intracranial hemorrhage

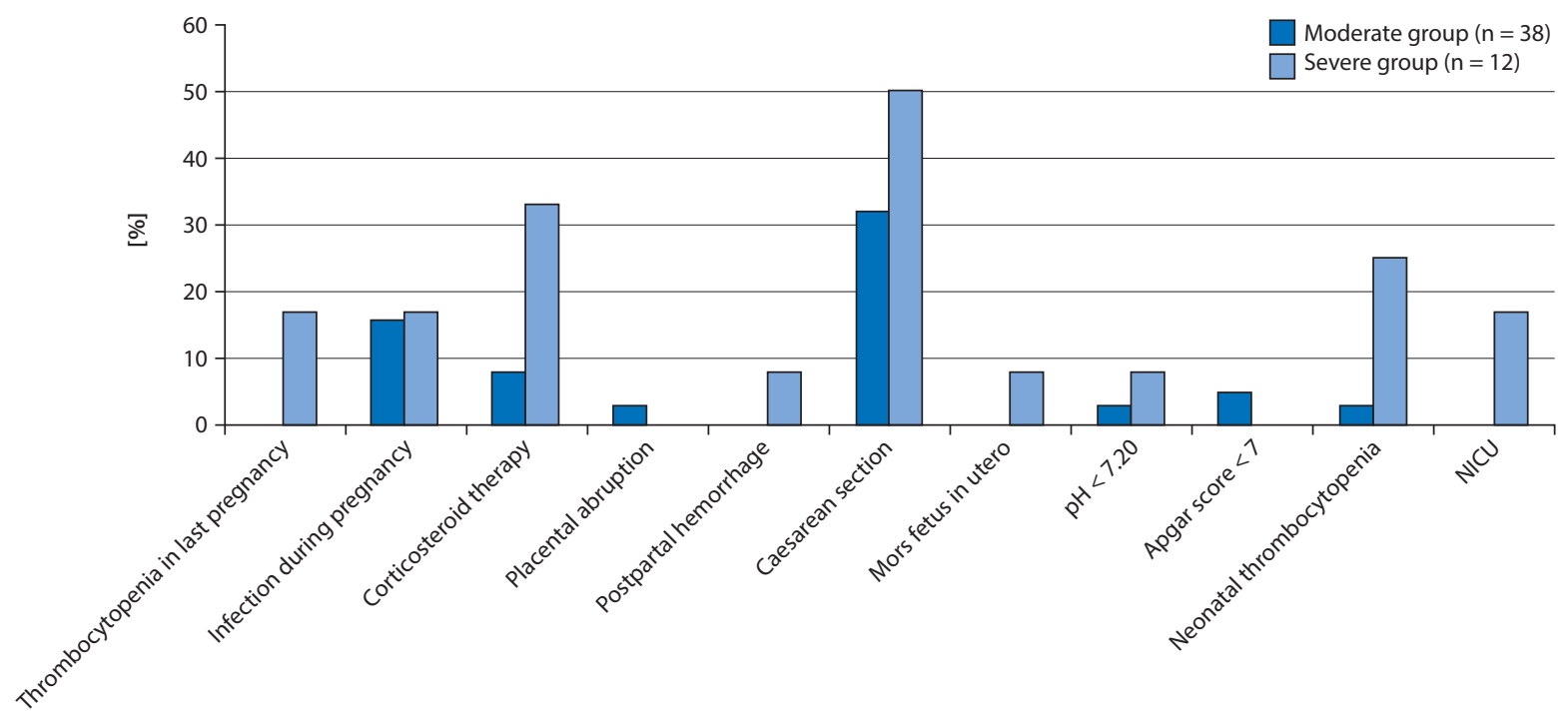

Figure 1. Pregnancy outcome with regard to severity of gestational thrombocytopenia 


\begin{tabular}{|l|c|c|c|}
\hline \multicolumn{3}{|l|}{ Table 3. The impact of severe gestational thrombocytopenia on the perinatal outcome and incidence of neonatal thrombocytopenia } \\
\hline Possible predictors & OR & $95 \% \mathrm{Cl}$ & P-value \\
\hline Maternal platelet count & 0.960 & $0.918-1.01$ & 0.071 \\
\hline Delivery mode & 0.698 & $0.369-19.73$ & 0.328 \\
\hline Gestational age & 0.778 & $0.441-1.37$ & 0.385 \\
\hline Birth weight & 0.999 & $0.998-1.01$ & 0.314 \\
\hline Apgar of < 7 at 1 min. & 15.667 & $1.09-224.37$ & 0.043 \\
\hline Neonatal acidosis (pH < 7.20) & 15 & $0.74-303.74$ & 0.078 \\
\hline Thrombocytopenia in previous pregnancy & 7.33 & $0.51-105.92$ & 0.144 \\
\hline Infection during pregnancy & 11 & $1.36-88.87$ & 0.24 \\
\hline
\end{tabular}

$\mathrm{OR}$ - odds ratio; $\mathrm{Cl}$ - confidence interval

thrombocytopenia in their previous pregnancies. Thrombocytopenia in previous pregnancy seems to be a valuable predictive factor for determining the severity of thrombocytopenia in the current pregnancy $(p=0.01)$. Corticosteroid therapy (oral prednisone) was used in 4 patients with severe thrombocytopenia (33.3\%, $p=0.027)$, and transfusion with packed platelet cells in 2 patients from the same group $(16.6 \%, p=0.01)$. We found significantly higher rate of neonatal thrombocytopenia $(p=0.041)$ in patients with severe gestational thrombocytopenia (Table 3).

We detected a significant influence of severe gestational thrombocytopenia on neonatal Apgar score at $1 \mathrm{~min}$. $(p=0.043)$ and high impact on neonatal acidosis $(p=0.078)$.

\section{DISCUSSION}

The most common cause of thrombocytopenia in pregnancy is gestational thrombocytopenia, which accounts for almost $75 \%$ of all cases [5]. The exact cause of gestational thrombocytopenia remains unclear, although it might be secondary to accelerated platelet consumption and increased plasma volume associated with pregnancy [8]. About $70-80 \%$ of thrombocytopenia cases in pregnancy are due to gestational thrombocytopenia, typically with a platelet count of $>70 \times 10^{9} / \mathrm{L}$, but a platelet count of $<70 \times 10^{9} / \mathrm{L}$ warrants an investigation for an alternative etiology [5, 7].

In our study group, 12 patients with severe gestational thrombocytopenia and a platelet count of $<50 \times 10^{9} / \mathrm{L}$ had negative tests for anti-platelet antibodies, so we classified them as severe gestational thrombocytopenia. Similar results were reported by Karim and Sacher [9], who documented the prevalence of severe thrombocytopenia in less than $0.1 \%$ pregnancies. As we enrolled only patients with a platelet count of $<100 \times 10^{9} / \mathrm{L}$ in our study group, the prevalence of gestational thrombocytopenia in our case was $0.4 \%$, whereas most studies accepted a platelet count of $<150 \times 10^{9} / \mathrm{L}$ as the cut-off for gestational thrombocytopenia, thus having higher prevalence [5]. This variance might be due to the exclusion of women with major systemic diseases, as well as those with gestational hypertensive diseases and other pregnancy-related disorders.

A platelet count of $<100 \times 10^{9} / \mathrm{L}$, the definition for thrombocytopenia adopted by the International Working Group [10], is observed in only $1 \%$ of pregnant women. Dwivedi et al. [11], observed a prevalence of $8.17 \%$, with $4.08 \%$ having severe thrombocytopenia in their study. The frequency of gestational thrombocytopenia in the largest series of consecutive women admitted for labor and delivery is $5 \%$.Comparing the neonatal outcome, we found no significant differences in the incidence of neonatal acidosis or Apgar scores between the groups. However, we observed mild neonatal thrombocytopenia $\left(>100 \times 10^{9} / \mathrm{L}\right)(\mathrm{p}<0.041)$ in 4 neonates $(8 \%)$ from our patients with gestational thrombocytopenia, with no other complications or need for admissions to the NICU. Ruggeri [12], reported a study of 41 pregnancies with gestational thrombocytopenia and found 2 infants with mild thrombocytopenia and 1 with severe thrombocytopenia, whereas none had clinically important bleeding. Burrows and Kelton [5], also found that moderate and severe fetal thrombocytopenia are a rare occurrence. In their prospective cohort study of 756 women with gestational thrombocytopenia, none of their patients and only 1 infant, who had congenital bone marrow dysfunction diagnosed later, had any bleeding complications [5].

In a study by Nagey et al. [13], 7 out of 730 pregnancies had platelet counts of $<150 \times 10^{9} / \mathrm{L}$, no neonate had a platelet count of $<100 \times 10^{9} / \mathrm{L}$, and no bleeding complications were observed. We found no specific possible predictors for this outcome that would be significant in the available literature (Table 3). There is no consistent correlation between the incidence of neonatal thrombocytopenia and the severity of maternal thrombocytopenia [5, 14, 15]. According to the earlier studies, history of thrombocytopenia in an older sibling is the best predictor of neonatal thrombocytopenia [16]. Regardless, other studies detected that severity of maternal thrombocytopenia is not a predictive factor for neonatal thrombocytopenia $[6,17]$. 
Thrombocytopenia is not usually considered as an indication for cesarean delivery and most studies recommend vaginal delivery, as long as there are no other obstetric indications present, even though neonates with severe thrombocytopenia may experience bleeding complications, especially intracranial hemorrhage, particularly as a consequence of head trauma during vaginal delivery. Ying-Hsuan et al. [18], analyzed 18,384 pregnancies and 787 of them (4.3\%) were complicated by thrombocytopenia. They found that thrombocytopenic women had a significantly higher rate of cesarean delivery as compared to their health peers. In our study, pregnancies with gestational thrombocytopenia had a higher general rate of cesarean delivery, although the difference was not statistically significant, and with no neonatal complications.

Several studies demonstrated that the risk of fetal intracranial hemorrhage in infants born to mothers with immune thrombocytopenia is also very low [5]. No studies have yet confirmed that the risk is increased during vaginal delivery. Provan et al. [19], suggest that the mode of delivery in pregnant patients with immune thrombocytopenia should be dictated solely by maternal indications, and the fetal platelet count not be routinely determined.

However, our study pointed that women with gestational thrombocytopenia at delivery had favorable perinatal outcomes, similarly to patients with a normal platelet count. A platelet count of $>50 \times 10^{9} / \mathrm{L}$ is generally considered safe for delivery (vaginal or cesarean) $[20,21]$. We found placental abruption in 1 patient from the study group (2\%) and 1 patient with severe postpartum hemorrhage (2\%), without neonatal complications, as compared to Dwivedi et al. [11], who detected $4.2 \%$ of postpartum hemorrhage and $2.4 \%$ of placental abruption cases in women with gestational thrombocytopenia. To the best of our knowledge, there are no long-term follow-up studies of women with gestational thrombocytopenia to determine if they have persistent thrombocytopenia.

It is known that system virus infections may cause thrombocytopenia by direct bone marrow suppression or increased peripheral platelet consumption. In our study, positive urine culture and vaginal bacterial infection were found in 8 patients from the study group (16\%). Parnas et al. [20], noted higher rates of Apgar scores of $<7$ at $5 \mathrm{~min}$. in infants born to mothers with thrombocytopenia versus healthy women $(p=0.001)$, intrauterine growth restriction $(p=0.003)$, and stillbirth $(p<0.001)$. Our results did not confirm their findings, probably because of our exclusion criteria.

Analyzing the possible risk factors for predicting neonatal thrombocytopenia in patients with severe gestational thrombocytopenia, we found maternal platelet count to be important but not significant (Table 3). We used corticosteroid therapy in 7 patients from the study group. Four patients
(33.3\%) had severe gestational thrombocytopenia. We had no need for intravenous immunoglobulin (IVIG). Kasai et al. [22], found that maternal treatment was required in $80 \%$ of immune thrombocytopenia subjects, but in none of the women with gestational thrombocytopenia, and the pregnancy outcomes were favorable in both groups, with no need for fetal treatment. Gernsheimer et al. [21], recommend the use of corticosteroids or IVIG if platelet count falls below 20 $-30 \times 10^{9} /$ L. Some studies consider the following as important predictors of an increased risk of neonatal thrombocytopenia: maternal history of splenectomy, maternal platelet count of $<50 \times 10^{9} / \mathrm{L}$ at some point during the pregnancy, and a previous pregnancy complicated with neonatal thrombocytopenia [23-26]. Noris et al. [27], performed a multicenter, retrospective study evaluating 339 pregnancies in 181 women. In their study, very early preterm deliveries, the lowest birth weight, the lowest Apgar scores, the greatest number with serious post-partum hemorrhage (>500 mL) were reported only in severely thrombocytopenic women. Also, the use of regional analgesia/anesthesia was the lowest, and the rate of cesarean sections was the highest in that group [28]. In their study, Aiyelaagabe et al. [29] reported 215 women with a platelet count of $\leq 120 \times 10^{9} / \mathrm{L}$ during pregnancy, and found that maternal platelet count did not correlate with either blood loss or neonatal platelet count.

According to our data, pregnancies with moderate or severe gestational thrombocytopenia, as compared to the control group, were not at a higher risk of cesarean section, intrauterine fetal death, preterm delivery, low Apgar scores, higher admission to the NICU rate, intracranial hemorrhage, neonatal death, or adverse maternal outcome.

\section{CONCLUSIONS}

Since gestational thrombocytopenia is usually mild or moderate, our data confirm that even severe gestational thrombocytopenia is not associated with adverse maternal or neonatal outcome. Also, moderate neonatal thrombocytopenia is more common in pregnancies complicated with severe gestational thrombocytopenia and the incidence of severe gestational thrombocytopenia is more frequent in patients with thrombocytopenia in previous pregnancy.

\section{REFERENCES}

1. Neunert C, Lim W, Crowther M, [et al.]. American Society of Hematology. The American Society of Hematology 2011 evidence-based practice guideline for immune thrombocytopenia. Blood. 2011, 117, 4190-4207.

2. Magann EF, Martin JN. Twelve steps to optical management of HELLP syndrome. Mississippi \& Tennessee classification systems for HELLP syndrome. Clin Obstet Gynecol. 1999, 42, 532-550.

3. Cunningham FG, Gant NF, Leveno KJ, [et al.]. Hematological disorders. Williams obstetrics, 21st ed. McGraw-Hill, New York 2001, 1307-1338.

4. Matthews JH, Benjamin S, Gill DS, Smith NA. Pregnancy-associated thrombocytopenia: definition, incidence and natural history. Acta Haematol. 1990, 84, 24-29. 
5. Burrows RF, Kelton JG. Fetal thrombocytopenia and its relation to 1aternal thrombocytopenia. N Engl J Med. 1993, 329, 1463-1466.

6. Sainio S, Kekomaki R, Riikonen S, Teramo K. Maternal thrombocytopenia: a population based study. Acta Obstet Gynecol Scand. 2000, 744-749.

7. Gill KK, Kelton JG. Management of idiopathic thrombocytopenic purpura in pregnancy. Semin Hematol. 2000, 37, 275-289.

8. McCrae KR. Thrombocytopenia in pregnancy. Hematology Am Soc Hematol Educ Program. 2010, 397-402.

9. Karim R, Sacher RA. Thrombocytopenia in pregnancy. Curr Hematolog Resp Mar. 2004, 3, 128-133.

10. Rodeghiero F, Stasi R, Gernsheimer T. [et al.]. Standardization of terminology, definitions and outcome criteria in immune thrombocytopenic purpura of adults and children: report from an international working group. Blood. 2009, 113, 2386-2393.

11. Dwivedi $P$, Puri M, Nigam Aagarwal K. Fetomaternal outcome in pregnancy with severe thrombocytopenia. Eur Rev Med Pharmacol Sci. 2012, 16, 1563-1566.

12. Ruggeri M, Schiavotto C, Castaman G, [et al.]. Gestational thrombocytopenia: A prospective study. Haematologica. 1997, 82, 341-342.

13. Nagey DS, Alger LS, Edelmann BB, [et al.]. Reacting appropriately to thrombocytopenia in pregnancy. South Med J. 1986, 79, 1385-1388.

14. Burrows RF, Kelton JG. Pregnancy in patients with idiopathic thrombocytopenic purpura: assessing the risks for the infant at delivery. Obstet Gynecol Surv. 1993, 48, 781-788.

15. Stavrou E., McCrae KR. Immune thrombocytopenia in pregnancy. Hematol Oncol Clin North Am. 2009, 23, 1299-1316.

16. Christiaens GC, Nieuwenhuis HK, Bussel JB. Comparison of platelet counts in first and second newborns of mothers with immune thrombocytopenic purpura. Obstet Gynecol. 1997, 90, 546-552.

17. Razafinstalama T, Coiffic J, Godin O, [et al.]. Percutaneous umbilical blood sampling in pregnant women with thrombocytopenia. Apropos of 35 cases. J Gynecol Obstet Biol Reprod. 2000, 29, 176-184.

18. Ying-Hsuan $\mathrm{L}$, Liang-Ming $\mathrm{L}, \mathrm{Ching}-\mathrm{Chang} \mathrm{H}$, [et al.]. Perinatal outcome in normal pregnant women with incidental thrombocytopenia at delivery. Taiwan J Obstet Gynecol. 2013, 52, 347-350.
19. Provan D, Stasi R, Newland AC, Blanchette VC, [et al.]. International consensus report on the investigation and management of primary immune thrombocytopenia. Blood. 2010, 115, 168-186.

20. Parnas M, Sheiner E, Schoam-Vardi I, [et al.]. Moderate to severe thrombocytopenia during pregnancy. Eur J Obstet Gynecol Reprod Biol. 2006, $128,163-168$.

21. Gernsheimer $\mathrm{T}$, James $\mathrm{AH}$, Stasi R. How I treat thrombocytopenia in pregnancy. Blood. 2013, 121, 38-47.

22. Kasai J, Aoki S, Kamiya N, [et al.]. Clinical features of gestational thrombocytopenia difficult to differentiate from immune thrombocytopenia diagnosed during pregnancy. J Obstet Gynaecol Res. 2015, 41, 44-49. doi: $10.1111 /$ jog. 12496 .

23. Webert KE, Mittal R, Sigouin C, [et al.]. A retrospective 11-year analysis of obstetric patients with idiopathic thrombocytopenic purpura. Blood. 2003, 102, 4306-4311.

24. Fujimura K, Harada Y, Fujimoto T, [et al.]. Nationwide study of idiopathic thrombocytopenic purpura in pregnant women and the clinical influence on neonates. Int J Hematol. 2002, 75, 426-433.

25. Payne SD, Resnik R, Moore TR, [et al.]. Maternal characteristics and risk of severe neonatal thrombocytopenia and intracranial hemorrhage in pregnancies complicated by autoimmune thrombocytopenia. Am J Obstet Gynecol. 1997, 177, 149-155.

26. Valat AS, Caulier MT, Devos P, [et al.]. Relationships between severe neonatal thrombocytopenia and maternal characteristics in pregnancies associated with autoimmune thrombocytopenia. Br J Haematol. 1998, 103, 397-401.

27. Noris P, Schlegel N, Klersy C, [et al.]. Analysis Of 339 Pregnancies In 181 Women With 13 Different Forms Of Inherited Thrombocytopenia. Haematologica. 2014, 99, 1387-1394. doi:10.3324/haematol.2014.105924.

28. Dikman D, Elstein D, Levi GS, [et al.]. Effect of thrombocytopenia on mode of analgesia/anesthesia and maternal and neonatal outcomes. J Matern Fetal Neonatal Med. 2014, 27, 597-602. doi: 10.3109/14767058.2013.836483.

29. Aiyelaagbe S, Hobson M , Byrd LM, Tower CL. Outcomes in women with thrombocytopenia in pregnancy. Arch Dis Child Fetal Neonatal. 2014, 99, A131. doi:10.1136/archdischild-2014-306576.381. 